Estudios Románicos, Volumen 29, 2020, pp. 121-134

ISSN: 0210-4911

eISSN: 1989-614X

DOI: https://doi.org/10.6018/ER.418951

\title{
LAS LOCUCIONES ADJETIVO-ADVERBIALES EN FRANCÉS Y EN ESPAÑOL, ¿PROBLEMA FRASEOLÓGICO O LEXICOGRÁFICO?
}

(Adjectival-adverbial locutions in French and Spanish, a phraseological or lexicographical problem?)

\author{
Hélène Cruz Modesti* \\ Universidad de La Laguna
}

\begin{abstract}
In the Hispanic and Francophone sphere, researchers have prioritized the locutions studies using a syntactic-functional criterion. However, this criterion, sometimes, lacks reliability, because certain units present the possibility of oscillation between two grammatical categories. This is the case, among others, of certain locutions with a prepositional phrase structure (en cruz, a ciegas, por defecto, de por vida, etc.), characterized by a syntactic duality that leads them to behave as adjective or adverbial locution. In this way, we may reflect on whether these are locutions that prototypically belong to the category of adverb or adjective and that, eventually, would work like the other or, on the contrary, the creation of a hybrid locutionary class (adjective-adverbial) is necessary. We also consider if the coining of this new subclass of locutions would be pertinent from a lexicographical perspective, especially bilingual Spanish-French.
\end{abstract}

Keywords: Translation; Linguistics; Phraseology; Lexicography; Locution; Dictionary.

Resumen: En los ámbitos hispánico y francófono, se ha priorizado la organización de las locuciones en función de un criterio sintáctico-funcional. Sin embargo, este criterio, en ocasiones, carece de fiabilidad, ya que ciertas unidades presentan la posibilidad de oscilar entre dos categorías gramaticales. Es el caso, entre otros, de ciertas locuciones con estructura de sintagma preposicional (en cruz, a ciegas, por defecto, de por $v i d a$, etc.), caracterizadas por una dualidad sintáctica que las lleva a comportarse como locuciones adjetivas o adverbiales. De este modo, nos planteamos si se trataría de locuciones que prototípicamente pertenecen a la categoría de adverbio o de adjetivo y que,

\footnotetext{
* Dirección para correspondencia: Hélène Cruz Modesti. Grupo de Investigación LexHis. Universidad de La Laguna. C/Anchieta n52, San Cristóbal de La Laguna, Tenerife (hcruzmodesti@gmail.com).
} 
eventualmente, funcionarían como otra o si, por el contrario, sería necesaria la creación de una clase locucional híbrida (adjetivo-adverbiales) es necesaria. Nos preguntamos también si la acuñación de esta nueva subclase de locuciones sería pertinente desde una perspectiva lexicográfica, en especial, bilingüe español-francés.

Palabras clave: Fraseología; Lexicografía; Traducción; Lingüística; Locución; Diccionario.

\section{Introducción}

Son numerosos los estudios que, desde hace varias décadas, vienen intentando organizar el espectro fraseológico, en función de diferentes concepciones -más o menos amplias - de la disciplina. Sin embargo, con independencia del lugar que se reserve a las locuciones -elemento central o subesfera fraseológica-, en todas estas propuestas de clasificación se observa una constante: el criterio en el que se basan es el de la función sintáctica que desempeñan en la oración, teniendo en consideración la categoría gramatical del núcleo del sintagma que ayuda a su clasificación (Casares 1950, Zuluaga 1980, Corpas Pastor 1996, García-Page 2008). En francés, al igual que sucede en español, las diferentes clasificaciones propuestas de la locution han seguido generalmente un criterio sintáctico-funcional. De hecho, Bally (1909) fue el primero en proponer la equivalencia entre locuciones y partes del discurso monoléxicas como criterio taxonómico, criterio que adoptarían otras clasificaciones, como la propuesta por el Dictionnaire de l'Académie française (DAF).

Sin embargo, la utilización histórica de este criterio sintáctico-funcional para organizar las locuciones no es del todo operante, ya que ciertas combinaciones presentan la posibilidad de oscilación entre, al menos, dos categorías. Es el caso de las locuciones adverbiales y adjetivas. Apunta ya Zuluaga que «no siempre es obvia la distinción y separación entre locuciones adnominales y adverbiales» (1980: 158). Tanto es así que, Castillo Carballo (2006: 22) plantea, para el español, la creación de una categoría independiente de locuciones adjetivo-adverbiales, que englobe unidades tales como: por ciento, a las claras, al contado, en cuclillas, bajo cuerda, en directo, a discreción, por etapas, por goleada, a granel, en jarras, etc., cuya naturaleza les permite asumir el rol de adjetivo o de adverbio en función del contexto.

En ocasiones, estas unidades [locuciones adjetivas y adverbiales] presentan comportamientos que dificultan su delimitación, en la medida en que en el discurso actualizan funciones adverbiales o adjetivales de acuerdo con el contexto. [...] Fundamentalmente, esto sucede con las que están constituidas por un grupo preposicional, que pueden desempeñar funciones de adjetivo o adverbios, según las relaciones que establezcan con el entorno lingüístico (Castillo Carballo 2015: 144-145).

Tradicionalmente, la consideración de estas locuciones, como locuciones adverbiales, o como adjetivas se rige principalmente por un principio de frecuencia. Sin embar- 
go, algunos estudios cuantitativos indican que la tendencia de estas unidades a funcionar como adverbio o adjetivo depende en gran medida de su forma. Por ejemplo, las locuciones construidas con la preposición de, como de cine, suelen corresponderse con mayor frecuencia con adjetivos. En cambio, las locuciones surgidas a partir de la preposición $a$, como a tope, suelen asumir mayoritariamente el rol de adverbio (Casti1lo Carballo 2015). Muchas otras unidades poliléxicas que no comienzan ni por «a» ni por «de» presentan la característica de poder asumir igualmente esta doble categoría y papel en el discurso. Por lo tanto, tal y como preconiza Castillo Carballo, cabría la posibilidad de proponer una clase locucional intermedia y tratar estas unidades como locuciones adjetivo-adverbiales, atendiendo a criterios de forma y de función.

A pesar de que, en francés, no existen estudios exhaustivos sobre el cambio de categoría de locuciones adverbiales a adjetivas y viceversa, creemos que se dan las condiciones propicias que permitirían proponer la creación de una categoría homóloga a la propuesta para el español. Este sería, el caso, entre otras, de la locución adverbial en croix, equivalente a la locución castellana en cruz. En ambas lenguas, estas unidades portan el mismo significado: 'referente a algo que tiene la forma de una cruz'. En español, la locución en cruz es susceptible de funcionar como adjetivo (1) o como adverbio (2).

(1) Una taza de café, bien caliente, y dos hojas de salvia en cruz 1 .

(2) Sangre seca te rodea en cruz con un solo pensamiento: ella.

De hecho, en el Diccionario de la lengua española (DLE) aparece recogida como locución adjetiva, con la advertencia de que también puede ser utilizada como locución adverbial. De igual modo, el Diccionario del español actual (DEA) también se hace eco de versatilidad funcional.

En francés, la locución en croix también se caracteriza por esta dualidad, de manera que podemos encontrar ejemplos en los que funciona como adjetivo (3) y otros en los que adquiere un valor adverbial (4):

(3) Notre pendentif en croix de baptême est d'une qualité remarquable héritée du savoir-faire des ateliers français.

(4) On y apercevait à peine la trace d'anciennes allées qui l'avaient jadis coupé en croix.

En lo que respecta a la manera de registrar la locución en croix en las obras lexicográficas de referencia, observamos que el Trésor de la langue française informatisé (TLFi) opta por clasificarla como una locución adverbial. Sin embargo, en el ejemplo que propone para ilustrar su definición «en forme de croix. Bras, jambes en croix», la locución funciona justamente como adjetivo. Lo mismo ocurre en el $D A F$, donde uno de los ejemplos proporcionados (5) refleja un claro valor adjetival, a pesar de que la combinación aparece marcada como locución adverbial.

1 A menos que se indique lo contrario, las fuentes de las que se han obtenido los ejemplos son el CORPES XXI, para el español, y diversas páginas web a través del administrador de corpus Sketch Engine, para el francés. 
(5) Deux couteaux en croix sur une table ont longtemps passé pour un mauvais présage.

Resulta curioso comprobar cómo en los grandes diccionarios generales de la lengua francesa no se hace alusión a la posible variación de categoría, y que incluyan ejemplos en los que las locuciones desempeñen una función no indicada. Lo confuso de esta situación, en principio, se podría resolver con la creación de la categoría locucional mixta adjetivo-adverbiales, como plantea Castillo Carballo (2015).

\section{Objetivos}

Frente a la evidencia, tanto en francés como en español, de la dualidad sintácticofuncional que caracteriza a ciertas locuciones con estructura de sintagma preposicional, cabe preguntarse: ¿estamos ante locuciones que prototípicamente se inscriben dentro de una categoría gramatical determinada (ya sea adjetivos o adverbios) y que funcionan eventualmente como otra o, al contrario, podríamos hablar, como se ha propuesto, de una clase locucional híbrida?, ¿en qué medida la acuñación de una nueva subclase de locuciones adjetivo-adverbiales sería pertinente desde el punto de vista fraseológico?, ¿y desde una perspectiva lexicográfica?

Con la intención de dar respuesta a estos interrogantes, comenzaremos nuestro estudio haciendo un repaso de lo que sucede en el caso de las monolexías que poseen un comportamiento análogo, es decir, que pueden funcionar como adjetivo o como adverbio. En este análisis, los conceptos de adverbialización, adjetivación y adjetividad ocuparán un lugar primordial. Un estudio cuantitativo basado en corpus reales servirá para confirmar o, al contrario, infirmar las hipótesis propuestas en nuestro análisis cualitativo, de manera que estaremos en posición de dilucidar, tomando como marco teórico la teoría de los prototipos (Kleiber 1995), la naturaleza de estas locuciones, en principio, duales. Antes de presentar las conclusiones de nuestro estudio, discutiremos la pertinencia de la creación de dicha categoría híbrida desde un punto de vista fraseológico y desde la óptica lexicográfica, en particular, de carácter bilingüe.

\section{3. ¿Más adjetivo o más adverbio?}

La Grammaire méthodique du français detecta un problema de definición de las clases sintácticas, tradicionalmente clasificadas a partir de criterios relacionados con las ideas que transmiten (critères notionnels), y alude al carácter flexible de estas categorías:

Ce type de classification est, en fait, dépourvu de tout pouvoir discriminant, puisque le nom, par exemple peut désigner, outre « les êtres vivants, les choses et les qualités » [...], toutes les notions qui sont également utilisées pour caractériser respectivement le verbe, l'adverbe et l'adjectif. [Ainsi,] si une même catégorie notionnelle peut se verbaliser dans plus d'une classe grammaticale et qu'une même classe grammaticale se prête à l'expression de plusieurs types de notions, cette absence 
d'univocité trouve une explication dans la spécificité des rôles syntactiques joués par chaque partie du discours et dans leurs contreparties interprétatives. (Riegel, Pellat y Rioul 1994: 118-119).

Así lo recalca también Alarcos Llorach (1987: 308) cuando señala que «esta cuestión del "calificar" o el "determinar" no es pertinente para el adjetivo, igual ocurre para el llamado adverbio» y concluye con la recomendación de que "hay que basarse en lo funcional». El problema es que, aun esgrimiendo la funcionalidad como criterio taxonómico, nos encontramos con casos de locuciones que, en principio, parecen manifestar un carácter dual. Para explicar esta duplicidad funcional, característica de ciertas unidades, investigadores como Bosque (2007: 48-52) proponen una hipótesis de duplicación de las categorías. En este mismo sentido, Kerleroux (1996: 101-108) habla de distorsion catégorielle. Esta autora reflexiona sobre las relaciones de posición de los lexemas en la frase, la jerarquía y la dependencia entre ellos, aparte de l'étiquetage catégoriel, y afirma que existe una disimetría entre la posición y el término que aparece en dicha posición: «il y a des séquences linguistiques qui sont analysables au double titre de leur composition interne en lexèmes, et des propriétés que leur procure l'occupation d'une position. Il y a des séquences linguistiques qui ne sont pas prises dans le treillis des positions» (1996: 22). Por lo tanto, concluye que, en realidad, existen dos elementos isomórficos, cada uno con una función específica. Por ejemplo: Mi colcha es violeta $($ violeta $=$ adjetivo $)$ vs. El violeta es un color $($ violeta $=$ sustantivo $)$.

Asimismo, el caso que nos ocupa en este artículo encuentra un homólogo en el ámbito monoléxico, ya que, como señala Noailly, el adverbio y el adjetivo comparten una relación lingüística muy estrecha: «l'adjectif a un rapport assez étroit avec l'adverbe. [...] il y a une relation analogique entre la modification du substantif par l'adjectif, et celle du verbe par l'adverbe, l'adjectif étant au nom ce que l'adverbe est au verbe» (1999: 148). Sin embargo, no siempre es clara la frontera entre adverbio y adjetivo.

\subsection{Adverbialización: adjetivos que funcionan como adverbios}

El fenómeno de la adverbialización es un ejemplo claro de cómo los adjetivos pueden adquirir valor adverbial. Este proceso se puede producir a través de la sufijación característica de los adverbios de modo construidos en -mente ${ }^{2}$ (6) o cuando un adjetivo permanece invariable y desempeña la función de adverbio (Hoyos 1993: 117) (7-8):

(6) Estaban felizmente casados ${ }^{3}$.

(7) María apunta alto.

(8) El equipo no juega limpio.

$2 \quad$ El sufijo -mente de estos adverbios de modo proviene de la palabra latina mentis, cuyo significado es 'forma o manera'. De este modo, un adverbio como felizmente, en realidad no se desliga completamente de su naturaleza adjetiva, ya que significa 'de manera feliz'. Este proceso explicaría, por ejemplo, que cuando este tipo de adverbios aparece en ocurrencia duplicada, el primero de ellos se pueda apocopar, p.ej. simple y llanamente. Sin embargo, este proceso no se puede dar en francés.

3 Los ejemplos del 6 al 12 están creados por la autora de este artículo. 
Este uso del adjetivo, en el que no acompaña a un verbo copulativo sino a uno predicativo, es característico de los denominados adjetivos adverbiales, es decir: «todas las formas adjetivas paralelas a los adjetivos calificativos ${ }^{4}$ que, sin embargo, no constituyen expresiones asignadoras de propiedades» (Bosque y Demonte 1999: 205).

Sin embargo, Luján (1980: 154) advierte que no todos los adjetivos que desempeñan la función de adverbio en una oración constituyen adjetivos adverbiales. De este modo, señala que los adjetivos adverbiales (9-10), contrariamente a los adverbios, están marcados por género y número, lo que evidencia su carácter adjetival.

(9) Los niños terminan cansados.

(10) La chica viajaba sola.

Como vemos, el adjetivo adverbial está declinado para ajustarse al sintagma nominal que acompaña. En realidad, se trata de una predicación secundaria, ya que en este tipo de oraciones se omite el verbo copulativo: Los niños terminaron cansados $=$ Los niños terminaron + los niños estaban cansados. No obstante, existen otros casos en los que la concordancia es imposible (11b y $12 \mathrm{~b})$ :
a. Las madres hablan bajo.
b. Las madres hablan *bajos.
a. Mi prima apunta alto.
b. Mi prima apunta *alta.

En estos ejemplos, no nos encontramos ante adjetivos adverbiales sino ante adverbios homónimos o isomorfos, ya que «un adverbio homónimo no puede presentar terminaciones de género o número [...] el resultado de tal manipulación es totalmente inaceptable» (Luján 1980: 155). Esta es la prueba más obvia para diferenciar ambas unidades ${ }^{5}$.

En francés, el uso de adjetivos que funcionen como adverbios también es recurrente. Noailly (1999: 148-149) explica las causas que llevan a emplear un adjetivo en lugar de utilizar el adverbio acabado en -mente. Si bien es cierto que esto pueda deberse a la inexistencia de un adverbio equivalente, p. ej. écrire compliqué // écrire *compliquement, en la mayoría de los casos estaríamos ante un rasgo característico del lenguaje familiar y oral: travailler dur adquiere más fuerza que travailler durement. Sin embargo, Guimier (1996: 68) apunta que los adverbios son más proclives a admitir sentidos figurados, mientras que los adjectifs invariés o adverbialisés tienden a preservar su sentido original, p.ej. parler bas y parler bassement de qqun, que significa hablar de forma inadecuada de los demás.

\footnotetext{
clasificadores.

Hay que destacar que Bosque ignora la existencia de otro tipo de adjetivos como intensificadores o

5 Para consultar más diferencias entre adjetivos adverbiales y adverbios homónimos, véase Luján (1980: 152-174).
} 


\subsection{Adjetivación: adverbios que funcionan como adjetivos}

Algunos adverbios monoléxicos pueden cumplir la función de adjetivo, p.ej. Gente bien, soy así, en francés, gens bien, je suis ainsi. Sin embargo, al permanecer invariables se alejan del adjetivo, como advierte Goes (1999: 218) «ils cadreraient plutôt dans une ressemblance de famille» ${ }^{6}$.

En el caso de las unidades poliléxicas, González Montero (1993: 518) afirma que «conjuntos formados por una base adjetiva y la colocación antepuesta de una preposición se han lexicalizado en locuciones adverbiales del tipo: a ciegas, a oscuras, a tontas y a locas, de nuevo, por último...». No obstante, no se trataría de un fenómeno de adverbialización, sino de transposición, de manera que una combinación Prep + S. Adj simplemente transita de una categoría a otra y se convierte en una locución adverbial. Además, muchas de estas locuciones eventualmente pueden funcionar como adjetivos, p. ej. cita a ciegas | rencontre à l'aveugle. En estos casos, sin embargo, sí que estaríamos ante una forma de adjetivación. Pero antes de abordar esta noción, es preciso establecer una distinción clara entre esta y el concepto de adjetividad.

En palabras de El Khamissy (2018: 43), «l'adjectivité se présente donc comme une notion généralisante regroupant tout mot ou partie du discours pouvant être employé adjectivement. Le processus même qui consiste à donner à un mot quelconque la valeur d'un adjectif est l'adjectivation», de manera que la adjetividad haría referencia a la probabilidad que tiene una palabra de ser empleada como adjetivo, mientras que la adjetivación aludiría al proceso por el que una unidad adquiere un valor adjetival. Por lo tanto, la alta o baja adjetividad de una palabra o locución podría ser indicador de la capacidad de oscilación de función entre adverbio y adjetivo.

Se desprende, pues, la necesidad de llevar a cabo un análisis, tanto en español como en francés, del grado de adjetividad de algunos elementos monoléxicos y locucionales que pueden funcionar indistintamente como adverbios o como adjetivos. Este estudio permitirá observar cuáles son las características comunes entre estos y los elementos adverbiales y adjetivales prototípicos, con el fin de dilucidar si las particularidades de las locuciones que nos ocupan en este artículo justifican la necesidad de crear una nueva categoría, o si, por el contrario, son combinaciones de naturaleza prototípicamente adverbial o adjetiva que, de forma puntual, admiten otra función.

\section{Análisis cualitativo}

La principal diferencia entre los adjetivos y los adverbios monoléxicos es su capacidad de aceptar flexión morfológica y, en menor medida, derivativa: mientras que los adjetivos admiten, por lo general, los morfemas de género y número, así como ciertos morfemas derivativos (p. ej. -ísimo, -ito, -ico, etc.), los adverbios, por su

$6 \quad$ Según Kleiber (1995: 151), la ressemblance de famille «caracteriza un conjunto de semejanzas entre los diferentes casos de una misma familia». No se trata de un prototipo, ya que no todos los miembros de una familia tienen que compartir todos los rasgos. Para ahondar en la diferencia entre prototipo y semejanza de familia, véase Kleiber (1995: 150-154). 
parte, son palabras, en principio, invariables. No obstante, ciertos adverbios, a pesar de que, efectivamente, no admiten flexiones morfológicas, en español ${ }^{7}$ aceptan sufijos derivativos, con independencia de que posean un equivalente isomorfo adjetival (13) o no $(14)^{8}$ :

(13) [...] la oye murmurar bajito antes de que la determinación brille en su voz.

(14) En esos momentos un tío de Facundo que vive cerquita les ayuda en las tareas del tambo.

La reticencia de las locuciones a aceptar cualquier morfema flexivo o derivativo parece de sobra justificada, ya que se caracterizan por un alto grado de fijación estructural. Sin embargo, esta invariabilidad, en muchos casos, es solo teórica, ya que locuciones como sano y salvo/sain et sauf admiten la flexión morfológica de género y número: sana y salva, saine et sauve, lo que confirma su carácter adjetival ${ }^{9}$. En el caso de las locuciones con esquema preposicional que nos ocupan, con independencia de que funcionen como adjetivos (15-16) ${ }^{10}$ o como adverbios (17-18), el criterio morfológicoflexivo no es operante:

(15) La mirada de soslayo/*de soslaya que me dirigió hizo que me derritiera por dentro.

(16) El corresponsal la miró de soslayo/ *de soslaya.

(17) Tout en marchant, elle regarde à la dérobée son petit ami de Zanarkand /*au dérobé

(18) Le Baiser à la dérobée, est une œuvre de Fragonard /*au dérobé

Por lo general, las locuciones tampoco aceptan morfemas derivativos. No obstante, en situación discursiva, encontramos casos de locuciones adjetivas o adverbiales que parecen contradecir esta tendencia, tanto en español (19-20) ${ }^{11}$ como en francés (21):

(19) [...] nuestros invitados a gustísimo disfrutando de cada momento.

(20) Bonita panera plastificada con estampado a rayitas de color azul celeste y blanco.

(21) Un assaut à l'aveuglette avec deux compagnons.

$7 \quad$ Este procedimiento expresivo, de uso frecuente en español, no es productivo en francés.

8 Estos dos ejemplos (13-14) en español se extraen de páginas webs a partir del administrador de corpus Sketch Engine.

9 Observemos que la locución sano y salvo puede funcionar como un adverbio isomorfo en oraciones como Aparecieron sanas y salvas este sábado cerca del mediodía o Plus de 30 personnes sont sorties saines et sauves de la boîte de nuit. En estos casos, al igual que las monolexías, admite su flexión morfológica, porque estamos ante una predicación secundaria: Aparecieron + estaban sanas y salvas; Elles sont sorties + elles étaient saines et sauves.

10 Estos dos ejemplos (15-16) en español se extraen de páginas webs a partir del administrador de corpus Sketch Engine.

11 Estos dos ejemplos (19-20) en español se extraen de páginas webs a partir del administrador de corpus Sketch Engine. 
Como ocurría en el caso de las monolexías, el francés ofrece una mayor reticencia a aceptar este tipo de morfemas derivativos, y cuando lo hace (à l'aveuglette, à mucette, en cachette, à la belle franquette), estamos ante fosilizaciones de otros estados de lengua que se han lexicalizado por completo. De hecho, las matizaciones apreciativas, axiológicas e hipocorísticas que, en español, suelen aportar los morfemas derivativos, en francés suelen realizarse a través de un procedimiento analítico, a través de la inserción de las lexías (trop, très, super, petit), como podemos apreciar en los siguientes ejemplos (2223):

(22) Mis en forme dans un moule, de forme boule, décoré de motifs à petits points rouges réalisés à la poire à engobe.

(23) Philippe et Sophie nous ont mis super à l'aise, ils sont super gentils.

Respecto a la sintaxis, la función del adjetivo monoléxico, como epíteto antepuesto o pospuesto, o atributo, así como la del adverbio, como modificador de un verbo, adjetivo u otro adverbio, está bien definida. En ese sentido, observamos que las locuciones adjetivo-adverbiales, al asumir las características sintácticas ya sea de un adverbio o de un adjetivo, carecen de cualquier rasgo diferenciador que pueda llevarnos a la creación de una nueva categoría mixta. Veamos registros de uso como adverbio (24-26) y como adjetivo (27-29):

(24) Estas divisiones de tareas pueden llegar a comprenderse en grandes obras, donde abundan los ayudantes o los materiales se compran a granel para varias tareas $[\ldots]$.

(25) La chaux, s'éventant à l'air, n'est pas expédiée en vrac; on la livre dans des sacs de 50 kilos ou mieux dans des barils garnis d'un sac ou d'une toile.

(26) Usaron a granel gas mostaza, Zyklon, ácido prúsico, fósforo, etc.

(27) [...] la expectativa continúa in crescendo en un evento que promete emociones a granel.

(28) La paille ou le fourrage en vrac sont encombrants et difficiles à manipuler.

(29) Arrivés en haut, derrière un lourd rideau noir, se trouvait un grand espace, des chaises, des miroirs, et des tissus en vrac.

Otra alternativa, tal y como proponemos a lo largo de este apartado, consistiría en decantarnos por la hipótesis que aboga por considerar estas locuciones como dos unidades isomorfas, cada una especializada en una función, y que serían el resultado de una distorsión. En este caso, habría que ver cuál sería la clase primigenia y cuál la que resulta de la distorsión. Para dilucidar esta cuestión a nivel sintáctico, creemos que es conveniente someter a nuestras locuciones a test transformacionales (véase tabla 1), lo que nos llevará a identificar con qué parte del discurso comparte más propiedades prototípicas. 
Tabla 1. Test transformacionales aplicados

\begin{tabular}{|c|c|c|c|c|c|}
\hline & $\begin{array}{c}\text { Adjetivo } \\
\text { (bonito) }\end{array}$ & $\begin{array}{c}\text { Locución } \\
\text { adjetiva } \\
(\text { de pacotilla })\end{array}$ & $\begin{array}{c}\text { Adverbio } \\
(\text { mucho })\end{array}$ & $\begin{array}{c}\text { Locución } \\
\text { adverbial } \\
\text { (a hurtadillas) }\end{array}$ & $\begin{array}{c}\text { Locución adjetivo- } \\
\text { adverbial } \\
(\text { en directo })\end{array}$ \\
\hline Atributo & $\checkmark$ & $\checkmark$ & $x$ & $x$ & $\checkmark$ \\
\hline Adjunto pospuesto & $\checkmark$ & $\checkmark$ & $\checkmark$ & $\checkmark$ & $\checkmark$ \\
\hline $\begin{array}{l}\text { Adjunto } \\
\text { antepuesto }^{12}\end{array}$ & $\checkmark$ & $x$ & $x$ & $x$ & $x$ \\
\hline Deslocalización & $x$ & $x$ & $x$ & $x$ & $x$ \\
\hline Enfatización & $\checkmark$ & $\checkmark$ & $\checkmark$ & $\checkmark$ & $\checkmark$ \\
\hline Coordinación & $\checkmark$ & $\checkmark$ & $\checkmark$ & $\checkmark$ & $\checkmark$ \\
\hline Relativización & $\checkmark$ & $\checkmark$ & $\checkmark$ & $\checkmark$ & $\checkmark$ \\
\hline Pronominalización & $\checkmark$ & $\checkmark$ & $x$ & $x$ & $x$ \\
\hline Exclamación & $\checkmark$ & $x$ & $x$ & $x$ & $x$ \\
\hline Gradación & $\checkmark$ & $x$ & $x$ & $x$ & $x$ \\
\hline
\end{tabular}

De este análisis se desprende que, de manera prototípica, el comportamiento de estas locuciones se acerca más al de los adverbios que al de los adjetivos, lo que nos conduce a identificar la función adverbial como primigenia y la adjetival como resultado de un proceso de distorsión. Es más, si observamos con detenimiento los casos en los que estas supuestas locuciones adjetivo-adverbiales funcionan como adjetivos, podemos comprobar que en la mayoría de casos podríamos hablar de la presencia de un participio omitido caracterizado por una locución adverbial, que toma la apariencia de locución adjetiva al aparecer en coalescencia con un sustantivo (30-31):

(30) Sin embargo, la instalación [hecha] por defecto nos deja el sistema preparado para trabajar únicamente con ASCII 7.

(31) Si vous êtes perdu et voulez qu' ExpressoWeb reprenne les paramètres [choisis] par défaut, il suffit de cliquer sur le bouton.

\section{Análisis cuantitativo}

Este análisis cuantitativo es un primer sondeo de los cien primeros testimonios que nos muestra el corpus ${ }^{13}$ de algunas de las posibles locuciones adjetivo-adverbiales. Así, vemos cómo el grado de representatividad nos muestra (véase tabla 2) que, de forma cuantitativa, estas locuciones son mayoritariamente adverbiales, aunque eventualmente funcionen como adjetivo.

12 La anteposición resulta en algunas combinaciones imposible: *realiza al por mayor vental*il fait en gros vente. En el caso de: mira en directo el programalil regarde en direct l'émission, se produce un ligero cambio de significado. En su empleo adverbial, no hay cambios: el programa se emitió en directolen directo se emitió el programa.

13 La identificación de la función sintáctica de las locuciones se ha llevado a cabo manualmente, puesto que la aplicación de reconocimiento automático de la categoría gramatical que ofrece el Sketch Engine presenta, por ahora, poca fiabilidad. 
Las locuciones adjetivo-adverbiales en francés y en español, ¿problema fraseológico o lexicográfico?

Tabla 2. Análisis cuantitativo

\begin{tabular}{|l|c|c|c|c|c|c|}
\hline & \multicolumn{2}{|c|}{ Función de adjetivo } & \multicolumn{2}{c|}{ Función de adverbio } & \multicolumn{2}{c|}{ Ocurrencias totales } \\
\cline { 2 - 7 } & ESP & FR & ESP & FR & ESP & FR \\
\hline A ciegas/à l'aveugle & 46 & 39 & 54 & 61 & 34813 & 6392 \\
\hline A escondidaslen cachette & 4 & 2 & 96 & 98 & 28127 & 7571 \\
\hline A granellen vrac & 67 & 84 & 16 & 33 & 37107 & 21222 \\
\hline De por vidalà vie & 46 & 53 & 54 & 47 & 80419 & 47368 \\
\hline De soslayolà la dérobée & 13 & 12 & 87 & 88 & 6176 & 910 \\
\hline En directolen direct & 65 & 48 & 35 & 52 & 311175 & 165790 \\
\hline Por defectolpar défaut & 53 & 34 & 47 & 66 & 114219 & 157774 \\
\hline
\end{tabular}

\section{Discusión}

A la vista de los resultados obtenidos en nuestros análisis cuantitativo y cualitativo, asumimos que la distorsión de categorías da lugar a una duplicación de combinaciones isomorfas, cada una con una función: una locución adjetiva y una locución adverbial. Sin embargo, según la Semántica de los prototipos de Kleiber (1995), estas unidades responden a un prototipo de base adverbial, del que se desprende un caso particular de carácter adjetivo.

De este modo, la acuñación de una nueva categoría fraseológica resultaría innecesaria. No negamos la posibilidad de que estas puedan agruparse en una subcategoría, pero nos parece poco operante, sobre todo porque, si tenemos en cuenta que otras muchas locuciones pueden también asumir distintos papeles sintácticos, el número de subclases aumentaría, sin ser indispensable desde un punto de vista estrictamente fraseológico. Una pregunta fundamental, sin embargo, queda todavía sin contestar: ¿cómo registraríamos estas unidades en obras de carácter lexicográfico?

A pesar de que en la fraseología este aspecto no sea trascendental, en lexicografía adquiere más importancia, ya que la marcación de las locuciones en los diccionarios no suele contemplar esta doble naturaleza, especialmente en los diccionarios bilingües, que contienen escasa información fraseológica. En este sentido, resulta interesante la visión de Mel'čuk (2006), quien propone una diferenciación entre la sintaxis profunda y la sintaxis de superficie. Las partes del discurso profundo son universales en casi todas las lenguas ${ }^{14} \mathrm{y}$, en cambio, la función sintáctica en la lengua particular haría referencia al discurso de superficie. En las locuciones, las partes del discurso profundo y de superficie se traducen en la estructura interna y en el empleo sintáctico. Así, el autor distingue las locuciones sintácticamente endocéntricas de las exocéntricas. En el primero de los casos, el más común, el núcleo de la locución nos indica su función. Así, por ejemplo, en pegar ojo o faire semblant la cabeza sintáctica es un verbo, por lo que estaríamos ante una locución verbal, que funciona como un verbo. No obstante, en el segundo caso, la función no está determinada por la estructura interna de la unidad, por lo que locuciones como por último o en douce, cuya cabeza sintáctica es una preposición, son

14 Verbo, sustantivo, adjetivo, adverbio y frase, clausal (traducido de clausatif). 
consideradas por Mel'čuk como locuciones preposicionales, pero que se emplean como un adverbio. En otras palabras, establece una distinción entre forma y función, que en las obras lexicográficas debería estar reflejada mediante una nomenclatura que permita acentuar esta dicotomía: loc. adjetiva (para la forma) vs. loc. adjetival (para la función) (2006: 20-23).

\section{Conclusiones}

Si adoptamos la teoría de Mel'čuk, todas estas locuciones potencialmente adjetivoadverbiales de esquema Prep $+\mathrm{S}$. Adj o $\mathrm{SN}$ serían locuciones preposicionales, ya que el núcleo es una preposición. Sin embargo, según su empleo, estaríamos ante un adjetivo o un adverbio. Esta diferenciación podría ser una solución satisfactoria para la lexicográfica monolingüe. Sin embargo, en la lexicografía bilingüe se pueden plantear otros problemas, ya que no siempre una unidad fraseológica se traduce por otra en la lengua destino.

El Diccionari descriptiu de la llengua catalana, uno de los más innovadores en la marcación fraseológica, propone una solución sencilla y práctica a este problema: la inclusión de las dos formas de las unidades como entradas diferenciadas. Siguiendo este modelo elaboramos un artículo lexicográfico bilingüe español-francés para una de las locuciones analizadas:

Vida $f .[\ldots]$

Locuciones

- De por vida loc. adj. À vie loc. adj. algo que dura todo el tiempo que alguien vive. Tiene una cicatriz de por vida. Chaque enfant est marqué à vie.

- De por vida loc. $a d v$. À vie loc. $a d v$. hacer algo que dure para siempre. Amó de por vida a la misma mujer. Il a été condamné à vie.

\section{BIBLIOGRAFÍA}

ALARCOS LLORACH, Emilio (1987): Estudios de gramática funcional del español. Madrid: Gredos.

BALLY, Charles (1951 [1909]): Traité de stylistique française. Génova: Libraire Georg \& Cie S.A, París: Libraire C. Klincksieck.

BOSQUE, Ignacio (2007): Las categorías gramaticales. Relaciones y diferencias. Madrid: Editorial Síntesis.

BOSQUE, Ignacio; DEMONTE, Violeta (1999): Gramática Descriptiva de la Lengua Española. Real Academia Española, Colección Nebrija y Bello. Madrid: Editorial Espasa Calpe.

CASARES, Julio (1950): Introducción a la lexicografía moderna. Madrid: Consejo Superior de Investigaciones Científicas.

CASTILLO CARBALLO, M. ${ }^{a}$ Auxiliadora (2006): «El universo fraseológico». Revista de lexicografía, 8, 25-41. 
CASTILlO CARBALlO, M. ${ }^{\mathrm{a}}$ Auxiliadora (2015): De la investigación fraseológica a las decisiones fraseográficas. Un estudio de interrelaciones. Vigo: Editorial Academia del Hispanismo.

CORPAS PASTOR, Gloria (1996): Manual de fraseología española. Madrid: Gredos.

CORPES XXI = Real academia española: Banco de datos (CORPES XXI) [en línea]. Corpus del Español del Siglo XXI (CORPES). Disponible en: <http://www. rae.es> [28/01/2020].

$D A F=$ Académie française (2011): Dictionnaire de l'Académie française (9. ${ }^{a}$ ed.). Versión electrónica. [https://www.dictionnaire-academie.fr; 30/01/2020].

$D E A=$ Seco, Manuel, Olimpia Andrés y Gabino Ramos (2000): Diccionario abreviado del español actual. Madrid: Aguilar Lexicografía.

$D L E=$ Real academia española y asociación de academias de la lengua española (2014): Diccionario de la lengua española (23. ${ }^{\mathrm{a}}$ ed.). Versión electrónica. [https://dle. rae.es/?w=diccionario; $29 / 01 / 2020]$.

EL KHAMISSY, Racha (2018): «De l'adjectif à l'adjectivité en français: regard croisé avec l'arabe». Studii de gramatică contrastivă, 30, 35-52.

GARCÍA-PAGE SÁNCHEZ, Mario (2008): Introducción a la fraseología española. Estudio de las locuciones. Barcelona: Anthropos.

GOES, Jan (1999) : L'adjectif. Entre nom et verbe. París, Bruselas: Champs linguistiques, Éditions Duculot.

GONZÁLEZ MONTERO, José Antonio (1993): «Interferencias entre el adjetivo y el adverbio de la gramática tradicional. Posibles soluciones» en Alfredo Rodríguez López-Vázquez (ed. lit.) Simposio "Didáctica de Lenguas y Culturas", 517-525.

GUIMIER, Claude (1996): Les adverbes du français. Le cas des adverbes en -ment. Paris: Ophrys.

HOYOS RODRÍGUEZ, Marta de (1993): «Un aspecto de la relación entre adjetivo y adverbio: adverbios y locuciones adverbiales. Implicaciones didácticas». $D i$ dáctica. Lengua Y Literatura, 5, 115.

INSTITUT DE ESTUDIS CATALANS. Diccionari descriptiu de la llengua catalana. Versión electrónica. [https://dcc.iec.cat/ddlc/index.asp; 3/02/2020].

KERLEROUX, Françoise (1996): La coupure invisible. Études de syntaxe et de morphologie. París: Presses Universitaires du Septentrion.

KLEIBER, Georges (1995): La Semántica de los prototipos. Categoría y sentido léxico. Madrid: Visor Libros. Traducción de Antonio Rodríguez Rodríguez.

LUJÁN, Marta (1980): Sintaxis y semántica del adjetivo. Madrid: Cátedra.

MEL'ČUK, Igor (2006): «Parties du discours et locutions». Bulletin de la Société de Linguistique de Paris, 101(1), 29-65.

NOAILLY, Michèle (1999): L'adjectif français. Paris: Ophrys.

RIEGEL, Martin ; PELLAT, Jean-Christophe ; RIOUL, René (1994): Grammaire méthodique du français. Paris: Quadrige, Presses Universitaires de France.

$T L F i=$ ATILF, CNRS, Université de Lorraine (1994): Trésor de la langue française informatisé. Versión electrónica. [http://atilf.atilf.fr; 30/01/2020]. 
ZULUAGA, Alberto (1980): Introducción al estudio de las expresiones fijas. Fráncfort del Meno: Peter D. Lang.

\section{PERFIL ACADÉMICO-PROFESIONAL}

Hélène Cruz Modesti se graduó en Estudios Francófonos Aplicados en la Universidad de La Laguna y recibió el premio extraordinario de Grado. Ha realizado el Máster de Traducción e Interculturalidad en la Universidad de Sevilla y el Máster de Formación del Profesorado Universitario de Educación Secundaria Obligatoria y Bachillerato, Formación Profesional y Enseñanzas de idiomas. Especialidad de lenguas modernas: Francés, por la Universidad Nacional de Educación a Distancia. Ha disfrutado de una Beca de colaboración durante el cuarto curso del Grado y de una beca de estudios de posgrado para realizar los estudios de máster concedida por el Cabildo de Tenerife. Actualmente, matriculada en el Programa de Doctorado en Artes y Humanidades de la Universidad de La Laguna, se interesa por la investigación en fraseología y lexicografía contrastiva español-francés. Además, forma parte del grupo de investigación LexHis de la Universidad de La Laguna.

Fecha de recepción: 18/03/2020

Fecha de aceptación: 22/04/2020 